
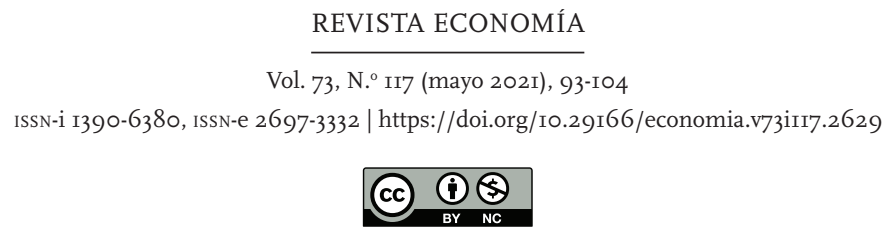

\title{
CONCENTRACIÓN Y TASAS DE INTERÉS EN EL SISTEMA FINANCIERO ECUATORIANO
}

CONCENTRATION AND INTEREST RATES IN THE ECUADORIAN FINANCIAL SYSTEM

\author{
PEDRO PÁEZ PÉREZ, ÓSCAR JARAMILLO VÁSCONEZ² Y RICHARD RAMÍREZ GONZÁLEZ² \\ ${ }^{1}$ Investigador independiente, ${ }^{2}$ Universidad Torcuato Di Tella (Argentina)
}

\author{
Recepción manuscrito: 30 de septiembre de 2020 \\ Aceptación versión final: $1 .^{\circ}$ de abril de 2021
}

\begin{abstract}
RESUMEN El paradigma neoclásico de la competencia impone en los mercados un modelo basado en la desregulación para conseguir el desarrollo que es definido como el mercado desregulado, en la práctica, esto solo provoca una mayor concentración. Con el objetivo de constatar los niveles de concentración en el sistema financiero ecuatoriano y sus consecuencias, se plantea un análisis de los indicadores de organización industrial y de las tasas de interés, considerando también la teoría de la competencia.
\end{abstract}

PALABRAS ClAVE Mercados financieros, tipos de interés, concentración de mercado, sistema financiero, Ecuador

\begin{abstract}
The neoclassical paradigm of competition imposes on the markets a model based on deregulation to achieve development that is defined as the deregulated market, in the real world, this only causes greater concentration. In order to determine the levels of concentration in the Ecuadorian financial system and its consequences, an analysis of the indicators of industrial organization and interest rates is proposed, also considering the theory of competition.

KEYWORDS Financial markets, interest rates, market concentration, financial system, Ecuador JEL CODES D43, E43, L13, L43
\end{abstract}

\section{INTRODUCCIÓN}

El Ecuador es una economía periférica integrada tardíamente a la dinámica del capital globalizado (Jaramillo y Ramírez, 2020). En los mercados globales existe una tendencia hacia la desregulación con el objetivo de cumplir las recomendaciones de la teoría neoclásica de la competencia, estas políticas ya han sido probadas en el país durante la última década del siglo xx 
con consecuencias negativas en el sistema económico, provocando entre otras cosas, la migración masiva de ecuatorianos. La desregulación responde a la estructura concentrada de los sectores financieros del país que terminan involucrándose en las decisiones públicas con serias consecuencias para el tejido productivo y la estabilidad financiera, por lo que es vital replantear el sistema. El objetivo de la investigación es constatar el nivel de concentración del sistema financiero ecuatoriano y sus efectos en la economía real, por lo cual se desarrolló un estudio de los principales indicadores de las empresas financieras del país y de los diferentes sectores que lo componen, usando indicadores de organización industrial.

\section{DISCUSIÓN}

Para entender la concentración del sistema financiero se debe abordar las corrientes neoclásicas de competencia que tienden por los aparentes beneficios de la desregulación aclamada como una «virtud y un avance respecto a la primitiva época anterior en la que el sector público ponía límites a la libertad del capital» (Weeks, 2009). Esta situación se refuerza en el ámbito social e incluso académico debido a «la propaganda del capital de que su libertad va en beneficio del interés general» (Weeks, 2009); esta máxima es considerada en la cultura globalizada de mercado como un principio fundamental e incluso con pretensiones de ley natural.

El objetivo detrás de la corriente de desregulación neoclásica es permitir a la ideología de la competencia reinar y asegurar la eficiencia en los mercados que terminarán por conducir al desarrollo (Hernández, 2010), concepto también polémico. La explicación de que esta sinrazón cale tan hondo en la sociedad globalizada de mercado e incluso pretenda competir en el ámbito cultural, es política, según Weeks (2009) «el mito neoclásico de la competencia es la defensa pseudotécnica del capital» y esconde las contradicciones bajo los principios «científicos» de la síntesis neoclásica de la economía.

Por esto, un análisis de la competencia requiere una ruptura metodológica con la economía neoclásica. Por un lado, Schumpeter (1997) llega a la conclusión que el sistema capitalista es dinámico y no estacionario. La esencia misma del capitalismo es ser discontinuo e impedir el desenvolvimiento de la competencia perfecta debido a las fallas de mercado, sin embargo, es esta misma característica la que permite que exista una competencia agresiva para desarrollar innovaciones tecnológicas, un proceso de destrucción creativa que termina relegando aquellas empresas que no puedan imitar o alcanzar dichas innovaciones. Por otro lado, Marx (1978) dice que la competencia y las crisis son inherentes al sistema capitalista, la competencia obliga a los capitalistas a desarrollar las fuerzas productivas que terminarían minando el proceso de acumulación capitalista.

Estas visiones de la competencia chocan con la concepción neoclásica donde la competencia es un mecanismo Deux ex machina para alcanzar el controvertido desarrollo. En la práctica, la desregulación de los mercados para alcanzar el desarrollo y la disminución del rol del Estado solo ahondan la desigualdad y generan concentración lo que impide la innovación y el progreso tecnológico además de mantener los precios altos. El sistema financiero no parece deslindarse de la producción real en cuanto a la dinámica de la competencia capitalista, aparte de la fragilidad intrínseca del sistema, estudiada por Minsky, ${ }^{1}$ el sistema financiero tiende a 
concentrarse de igual manera, la desregulación y la globalización afectan la dinámica del sistema de forma especial debido a que el juego de centralización de capital se profundiza por capitales financieros internacionales consolidados que terminan en muchos casos siendo los acreedores de naciones enteras, la desregulación también ha permitido la fuga de capitales a los centros de gravitación financiera del mundo o a los paraísos fiscales.

De hecho, un mejor rendimiento del sector financiero parece mostrar una relación estrecha con el desarrollo económico (Levine, 1997) mejorando la productividad a través de la movilización de recursos para financiar proyectos y la evaluación de los posibles empresarios (King y Levine, 1993), diversificando el riesgo de las actividades innovadoras (Rochet y Freixas, 1999) y minimizando la brecha entre la escasez de liquidez de algunos agentes y el exceso de liquidez de otros (DeYoung y Rice, 2004).

Por el contrario, la concentración del sistema financiero afecta también al sector real, «[1] a evidencia también parece indicar que el poder de mercado bancario puede representar una barrera financiera para la entrada en industrias no financieras» (Cetorelli, 2006), y viceversa, «[1]a evidencia empírica también sugiere que la desregulación procompetitiva de la industria bancaria [...] ha contribuido a reducir el tamaño medio de las empresas de los sectores no financieros» ${ }^{2}$ (Cetorelli, 2006).

En otro estudio sobre las desregulaciones del sistema bancario, «[u]n análisis de las medidas de concentración estructural ${ }^{3}$ indican que la concentración del sector bancario aumentó en la mayoría de los países europeos entre 1997 y 2003» (Casu y Girardone, 2006), los años donde se implementó la desregulación, es decir, tanto el sector financiero como el sector real son afectados negativamente por la desregulación de los mercados que lleva a la concentración.

\section{EL CASO DE AMÉRICA LATINA/ECUADOR}

En cuanto a América Latina y Ecuador, la situación no es diferente, los resultados de la desregulación han llevado a un capitalismo oligopólico que impide el ansiado desarrollo capitalista. Lo que para Jaramillo y Ramírez (2020) parece contradecir la ideología de mercado e incluso las imbatibles teorías económicas neoclásicas de la competencia. Para Morandé (2016), las reformas beneficiaron a los grupos sociales dominantes, dando paso a la formación de monopolios, oligopolios y grupos económicos, que dicho sea de paso, se unieron a otros ya existentes desde la época colonial. Para Durand (2020), es la época de adopción de un nuevo paradigma económico donde se fortalece a los privados y, dentro de ellos, a las grandes empresas locales y las multinacionales.

En el sistema bancario, el subsistema más dominante dentro del sistema financiero (Vera y Titelman, 2013) la historia parece ser la misma. Marichal y Gambi (2017) señalan que los bancos más grandes de América Latina de la época contemporánea también se encuentran entre las empresas capitalistas más antiguas e importantes de la región, y se han caracterizado a lo largo de los años como un sector altamente concentrado. Esto también se puede ver en las fortunas que la banca suele alcanzar en Latinoamérica.

En Ecuador, la realidad no es diferente, el sistema financiero del país avanzó desde «la extraordinaria alza del precio del cacao [...] que creó las condiciones para el establecimiento de 
un banco» (Miño Grijalva, 2008) hasta «los primeros años del siglo xxi y sus tendencias desreguladoras que llevaron desde el año 2000 a valores altos de los diferentes ratios de concentración tanto para cartera y depósitos» (Camino, Uzcátegui y Moran, 2017). «En el Ecuador existe una premisa sobre un sector bancario con características oligopólicas y con un alto poder de mercado, ya que existen 4 bancos considerados grandes» señalan (Camino, Uzcátegui y Morán, 2017); este nivel de concentración mantiene las tasas de interés elevadas lo que impide el correcto auspicio a la industria. La concentración también está relacionada con las utilidades.

Existe también evidencia de que la competencia en el sector financiero puede afectar la estabilidad del mismo de formas diferentes, para el caso del país hay que considerar que «la competencia sin control puede conducir a la fragilidad en un entorno institucional débil» (Beck, 2008). Considerando la extrema situación cambiaria del país por la dolarización, se debe considerar que altos niveles de concentración del sector financiero nacional pueda traer serias dificultades.

En cuanto a la concentración del sistema financiero ecuatoriano, la teoría económica afirma que las tasas de interés pueden subir, lo que tiene consecuencias negativas en cuanto al financiamiento del sector real y el auspicio a la innovación especialmente para ciertas provincias del país, pero hablar de un mercado financiero más competitivo también parece indicar una menor estabilidad financiera.

En el Ecuador para el periodo 2003-2016 se comprobó que la relación entre competencia y estabilidad medida por el riego de crédito es estrictamente negativa, es decir a mayor competencia bancaria se presentara una menor estabilidad. Puesto que la competencia entre bancos tiende a reducir las rentas de las instituciones financieras, lo que incentiva a los bancos a tomar mayores riesgos. (Pozo, 2018)

La aparente estabilidad financiera que provocaría un sistema financiero concentrado puede incluso ser mayor aumentando la competencia entre los sectores, como el auspicio a la economía popular y solidaria donde la concentración es menor y los créditos más asequibles para diferentes provincias del país y diferentes sectores productivos.

El mercado cooperativo ecuatoriano presenta una estructura de mercado competitiva, pues el IHH se encuentra por debajo de 1000 en las tres variables analizadas, el ID indica un bajo nivel de dominancia en las variables activos y cartera de crédito y moderado en los depósitos. (Regalado y Espinoza, 2018).

Lo que no ocurre en los bancos privados los cuales mantienen un alto índice de concentración de mercado que lleva a una alta rentabilidad. Vera-Gilces, Camino-Mogro, OrdeñanaRodríguez y Cornejo-Marcos (2019) señalan que la rentabilidad de los bancos privados en el Ecuador tiene una relación positiva con el ratio de capital, el poder de mercado, la competencia, el índice del dólar y la exposición al riesgo cambiario indirecto.

[...] Las instituciones supervisoras del sector financiero deberían endurecer las políticas centradas en el poder de mercado, especialmente en este sector, ya que un mayor poder de mercado podría estar relacionado con mayores márgenes financieros. (Vera-Gilces, Camino-Mogro, Ordeñana-Rodríguez y Cornejo-Marcos, 2019) 
A partir de estas consideraciones, se planteó el análisis de los indicadores de organización industrial y las tasas de interés buscando entender el sistema financiero ecuatoriano y cómo sus características afectan la economía del país.

\section{METODOLOGÍA}

Para el estudio se utilizaron las bases de datos de la Superintendencia de Bancos del Ecuador, donde constan todas las empresas del sistema financiero inscritas en esta desde 2002 hasta 2018.

En la Superintendencia de Bancos existe también la variable sector que se refiere a la subdivisión que la entidad clasifica para las diferentes empresas y son la unidad sobre la cual se establecieron los indicadores de concentración. Los sectores son banca privada, banca pública, compañías de titularización hipotecaria, mutualistas, oficinas subsidiarias en el exterior, sistemas generales de depósitos, sistemas de casas de cambio, sistemas de grupos financieros, sociedades financieras y tarjetas de crédito.

Hay que considerar que para el análisis de concentración es necesario clasificar el mercado con un enfoque de sustitución, sin embargo, ya que «la interpretación de las medidas estructurales de mercado como el porcentaje de mercado de las 4 mayores empresas y el índice Herfindahl-Hirschman se complica enormemente por una variedad de factores, empezando por el hecho de que cualquier estructura de este tipo es endógena» (Pepall, Richards y Norman, 2014). La clasificación sectorial de la superintendencia si bien da un buen marco para clasificar las empresas, por lo general se basa en técnicas similares y no en una medida de sustitución, razonamiento utilizado para la concentración de mercado. Ya que este estudio se basa en la concentración de variables contables de otro tipo, el criterio de técnicas similares tiende a ser más fuerte que el criterio de sustitución, ya que en cuanto a la producción de las empresas las categorías deben ser comparables, por esto se decidió utilizar la misma clasificación sectorial de la Superintendencia de Bancos para clasificar el mercado y los indicadores.

Las tasas de interés ponderadas efectivas por subsistema y sistema financiero en su totalidad corresponden a los boletines mensuales emitidos por el Banco Central del Ecuador. A nivel provincial se calculó un promedio simple con las tasas de interés efectivas por ausencia de las ponderaciones para cada tipo de crédito. La información del spread del sistema financiero total de los países, excepto Ecuador, fue tomado del Banco Mundial con la información del año más reciente. La cartera y volumen de crédito de los bancos privados fue proporcionada por los boletines mensuales de la Superintendencia de Bancos, y en el caso del crédito per cápita se tomó la información de la población de cada provincia en base a las proyecciones poblacionales del Instituto Nacional de Estadísticas y Censos (INEC).

\section{INDICADORES}

Se decidió utilizar el índice Herfindahl Hirschmann (HHI) y el porcentaje de la participación de las cuatro mayores empresas por cada sector $(4 \mathrm{M}),{ }^{4}$ para cada empresa, año y variable. El índice HHI es una medida común para medir la concentración de mercado. Es calculado elevando al cuadrado la proporción que cada empresa tiene del mercado y sumando los resultados, está en un rango entre o y 1. 


$$
H H I=\sum_{n=1}^{i} S_{n}^{2}
$$

4M responde a la suma de los cuatro mayores porcentajes de participación del mercado de las empresas.

$$
4 m=\sum_{n=1}^{4} s_{n} \text { donde } n_{1} \ldots n_{4} \text { son los cuatro mayores }
$$

Los indicadores asignan valores diferentes de concentración a las clasificaciones sectoriales, esto se debe a la naturaleza matemática de cada indicador, 4M es un indicador bastante lineal, mientras que HHI es un indicador exponencial que pondera los valores y polariza la clasificación. 4M por el contrario al ser un indicador lineal asigna el mismo peso a cada empresa y toma el porcentaje simple de las cuatro mayores empresas del total del sector, sin embargo, «las dos medidas de concentración industrial están muy relacionadas, lo que implica que cada una de ellas ofrece aproximadamente la misma descripción de la estructura de una industria» (Pepall, Richards y Norman, 2014).

\section{RESULTADOS}

Para evaluar la concentración en el sector financiero, es necesario considerar el número de empresas que tradicionalmente se ha mantenido estable debido a la presencia de barreras de entrada al mercado típicas de la rama del negocio, pero también a la dinámica competitiva oligopólica. En relación a los bancos privados se puede identificar tres ciclos diferentes en cuanto al número de competidores: el primero del 2002 al 2012 donde el número de bancos aumenta de 22 a 26; el segundo, se establece en el período 2013-2015 donde la disminución del número de competidores es igual al aumento del primer ciclo; el tercer período se establece en 20162018 con la entrada de dos competidores, alcanzando 24 empresas en el sector para el 2018.

La banca pública, las mutualistas y las sociedades financieras se han mantenido más estables, de 2002 a 2018: la banca pública se redujo de seis a tres instituciones; las mutualistas de siete a cuatro y las sociedades financieras de once instituciones a cinco.

Existen cuatro tipos de instituciones más que conforman el sistema financiero: almaceneras, casas de cambio, compañías de titularización hipotecaria, tarjetas de crédito y las entidades operativas en el exterior. Estos tipos de instituciones constituyen un número reducido de empresas, para el año 2018 tan solo 11 en estos sectores. Existen también mercados sumamente reducidos como casas de cambio, o incipientes como tarjetas de crédito, cuya particularidad determina que el número de instituciones sea muy reducido.

Sin importar el sector, el número de instituciones financieras ha permanecido bajo en comparación con otras actividades del sector real durante el periodo estudiado. Los sistemas financieros tienden a ser sectores difíciles de entrar debido a las fuertes inversiones que suponen y a la cantidad de requisitos que se deben cumplir, sin embargo, en Ecuador la cantidad de competidores en dicho sector parece ser el reflejo de una estructura oligopólica concentrada que 
Figura 1. Bancos privados: Evolución del HHI y 4M por variable (2002-2018)

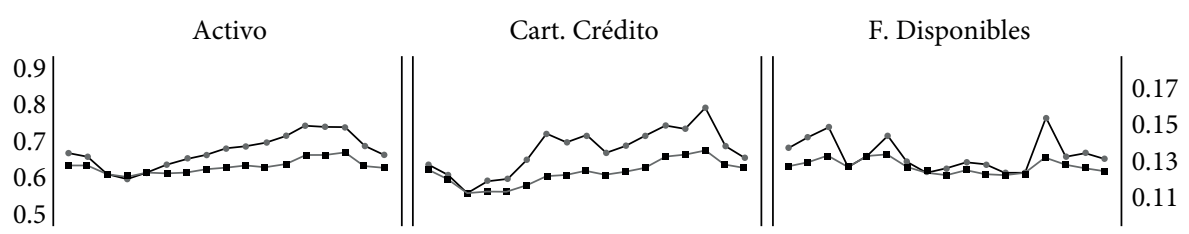

Gasto en personal

Impuestos

Ingresos

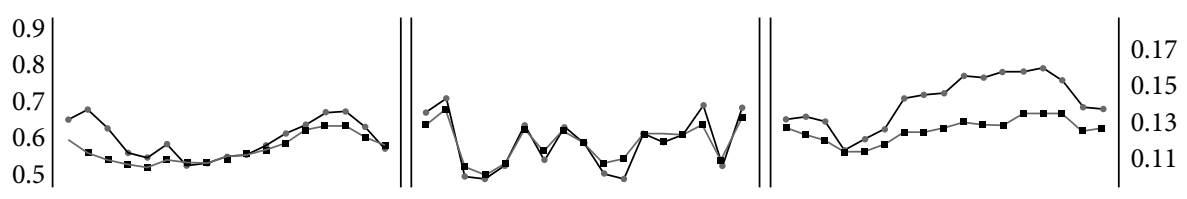

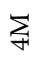
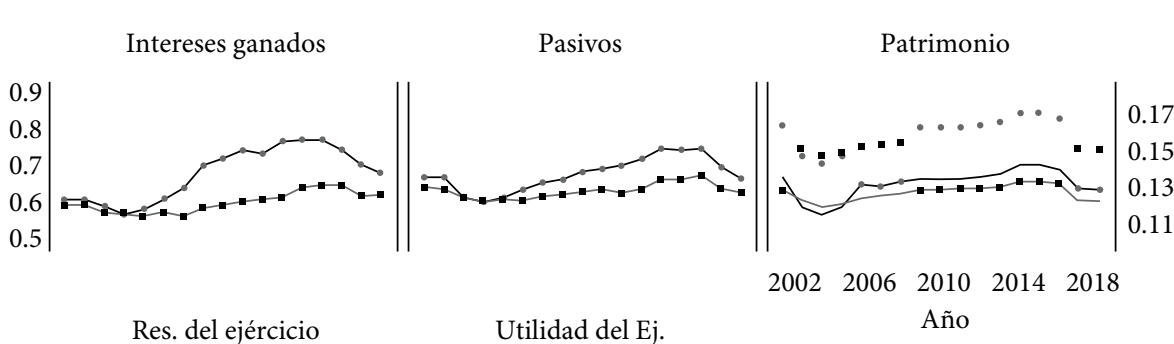

표

Res. del ejércicio

Utilidad del Ej.

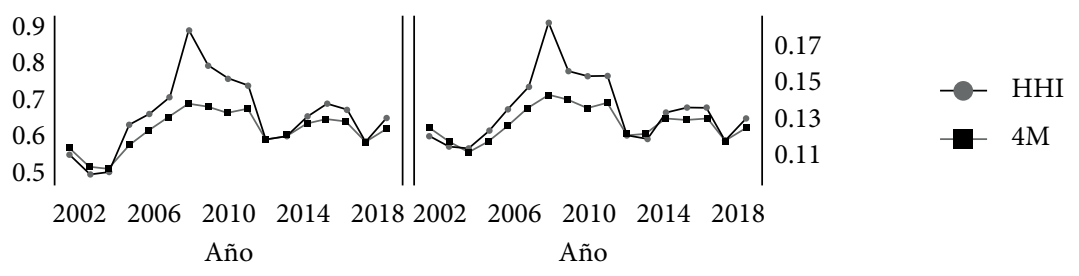

Fuente: Superintendencia de Bancos del Ecuador.

incluso puede ser mayor si se considera las relaciones entre ellos, sobre todo en el sector bancario el cual se analizará más a profundidad.

En la figura 1 se puede ver el nivel de concentración de los bancos privados por los indicadores HHI y 4M para cada una de las variables contables consideradas, nótese que todas las variables crecen en el tiempo, para mantenerse estables en el periodo siguiente (ver Figura 1). Desde el año 2002, existe una alta concentración en ingresos e intereses ganados, y la tendencia ha aumentado a partir del año 2007 hasta el año 2015, donde empieza a descender para el índice Hнi y para 4M. Nótese, ademá,s que los coeficientes oscilan entre 0.5 y 0.7 para 4M, mientras que HHI arroja coeficientes entre 0.1 y 0.2 pero presenta el mismo movimiento.

Los bancos que dominan el sector no solo controlan la mayor parte de ingresos, también se hacen con la mayor parte de los intereses ganados, la utilidad del ejercicio y los pasivos, si 
Figura 2. Tasa de interés ponderadas efectivas por sector 2015-2019

Tasas de Interés

$20.0 \%$

$15.0 \%$

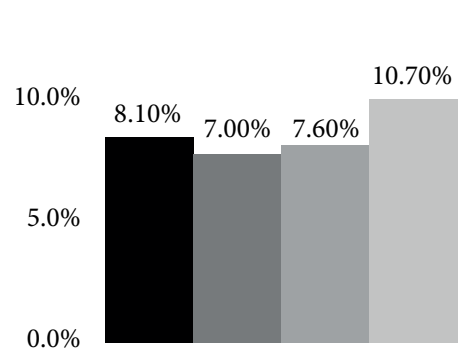

Spread
$18.10 \%$

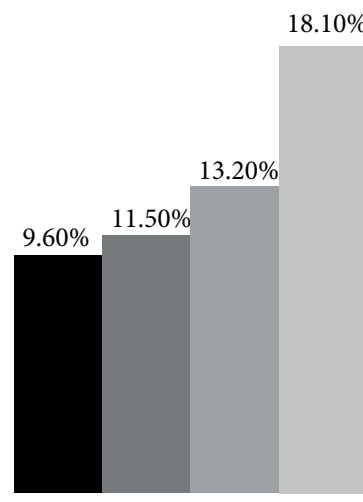

Tasa activa efectiva

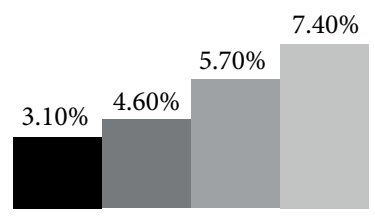

Tasa pasiva efectiva

Banca pública $\square$ Bancos privados Mutualista $\square$ Coop. Ahorro y crédito

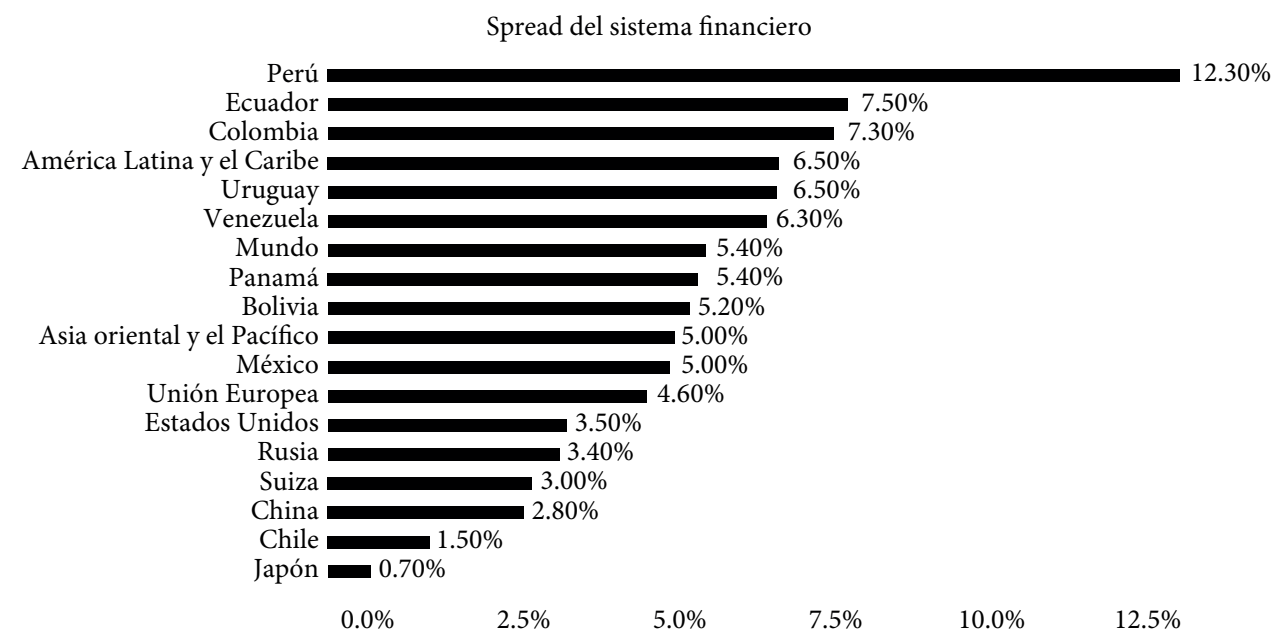

Fuente: Banco Mundial y Banco Central del Ecuador.

se mide la concentración a través de HHI. 4M da como resultado una alta concentración en la utilidad del ejercicio, activos, fondos disponibles y pasivos. Además, los cuatro bancos más grandes capturan el 61\% de la cartera de crédito lo que respalda lo encontrado por Camino, Uzcátegui y Moran (2017). Algo importante a mencionar es que las dos variables menos concentradas son los impuestos y el gasto de personal. Es decir, las dos variables que de una u otra forma regresan a la sociedad son las menos concentradas por los bancos. 
Figura 3. Bancos privados: crédito per cápita y tasa de interés activa efectiva por provincia (2015-2019)
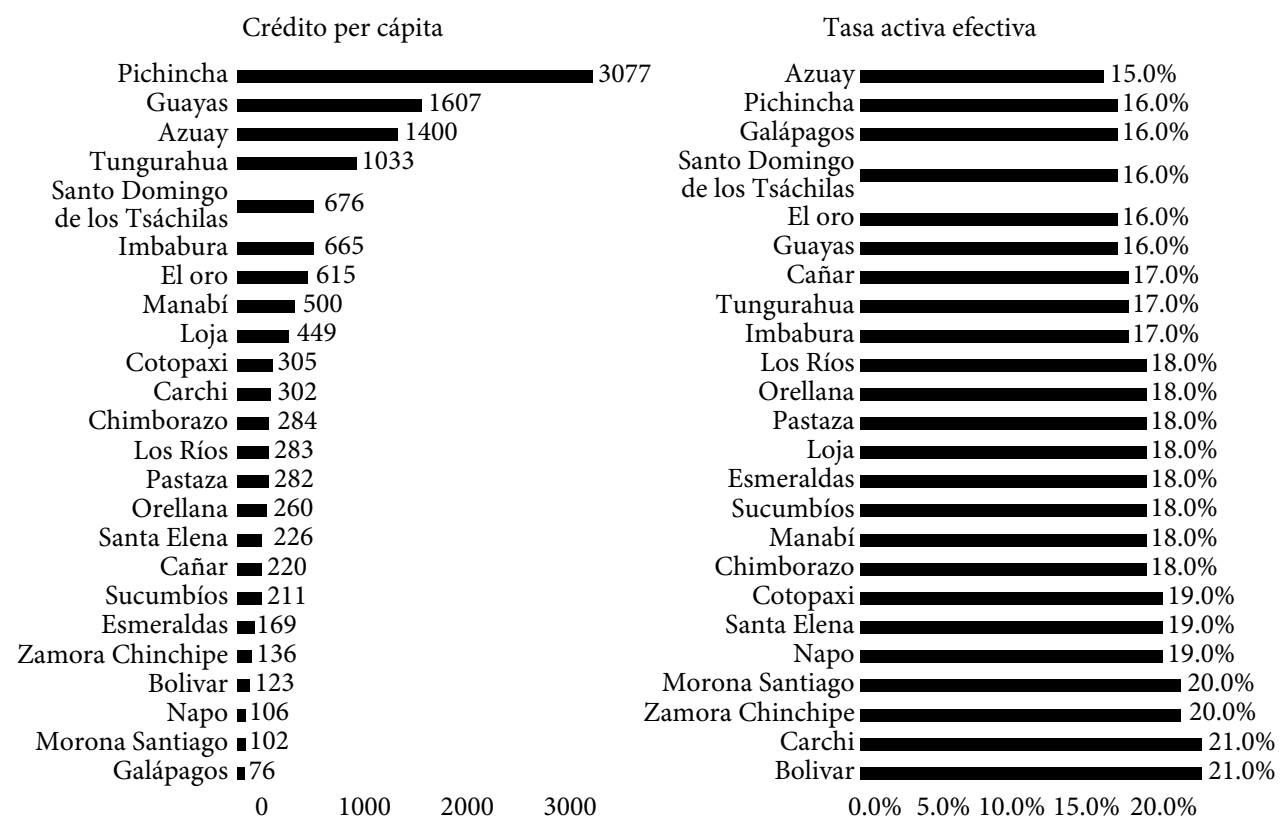

Fuente: Superintendencia de Bancos del Ecuador.

Los indicadores también muestran algunas contradicciones, la concentración medida a través de HHI de las diferentes variables tienden a ser más altas durante los años $2007 \mathrm{al}$ 2015, la concentración de utilidades tiene un pico en el 2008 y no se corresponde con los ingresos, que presentan una mayor concentración en el año 2014. Existe también una alta concentración de activo y pasivo, las cuales han presentado un incremento a partir del año 2005 hasta el año 2016, a partir de dicho año la concentración comienza a disminuir. La comparación de la concentración de impuesto, ingresos y utilidades muestra no solo cómo las utilidades parecen no estar relacionadas con los ingresos, sino también como los impuestos parece no estar relacionado con ninguna de ellas, lo que empíricamente no se esperaría.

El índice 4M indica un escenario similar del sistema bancario privado del país. Los cuatro bancos más grandes controlan el $62 \%$ del total de activos e ingresos y los 20 bancos restantes apenas concentran el $38 \%$. Se puede ver también que la concentración de las utilidades de los cuatro bancos más grandes disminuye, a partir del 2008, a pesar que la concentración de ingresos de estos mismos bancos aumenta. La concentración de impuestos en el 2018 cayó, a pesar que fue el año donde los cuatro bancos más grandes concentraron la mayor parte de las utilidades. Lo cual es poco usual.

Todo esto se expresa en las tasas de interés y en el impacto que tiene el sistema bancario en la sociedad. En la figura 2 se puede ver las tasas de interés según sector del sistema financiero 
y una comparación del spread a nivel internacional, nótese que la tasa activa varía por sector financiero, siendo más alta para las cooperativas de ahorro y crédito, según Asobanca (2019) la tasa activa debe cubrir la prima de riesgo, los costos operativos, los costos de fondeo, impuestos, contribuciones y las ganancias (ver Figura 2). Por lo que es de esperar que las coperativas tengan más costos y riesgos que cubrir, la banca privada, sin embargo, debería ser capáz de reducir costos por economías de escala y de diversificar el riesgo, sin embargo el spread financiero es apenas diferente de las mutualistas. Nótese también que el Ecuador se encuentra entre los países con un más alto spread de todo el sistema financiero a nivel internacional.

En cuanto al sector de los bancos privados, se observa en las ilustraciones 3 y 4 que los créditos se concentran en las provincias de Pichincha, Guayas y Azuay, evidentemente debido al movimiento comercial, al mismo tiempo la tasa activa es menor en estas provincias y mayor en las provincias donde la concentración del crédito es menor (ver Figuras 3 y 4). Finalmente, en la figura 4 se aprecia el destino del crédito otorgado por la banca privada. Como se puede ver la mayor cantidad de créditos son los comerciales con una tendencia negativa, mientras que los créditos de consumo no solo representan un porcentaje alto de los créditos sino que crecen durante el periodo de análisis.

\section{CONCLUSIONES Y ALTERNATIVAS}

De los resultados se verifica la hipótesis de que el sistema financiero ecuatoriano presenta un nivel de concentración entre alto y moderado en todos los sectores según los indicadores HHI y 4M. Este nivel de concentración no necesariamente está relacionado con consecuencias negativas para la economía según la literatura revisada, sin embargo, la concentración puede llevar a tasas de interés elevadas, como sucede en el Ecuador con el segundo spread bancario más alto de la región, inclusive por encima del promedio de América Latina y el Caribe.

Hay que considerar también que el Ecuador representa un caso particular debido a la dolarización, el entorno institucional débil y la injerencia de grupos financieros en el poder político. Estas características pueden profundizar los efectos negativos de la concentración y deben ser profundizadas en estudios posteriores.

De acuerdo a las ilustraciones, el crédito de la banca privada está ampliamente concentrado en las principales provincias del país, esto, por supuesto, refleja los costos y la prima de riesgo presente en las diferentes empresas y es un argumento a favor de las cooperativas de crédito que pueden absorber la demanda de las provincias olvidadas por la banca privada, también refleja una completa desinversión financiera al menos en las provincias más descuidadas y gerencias tradicionales de la banca privada indispuesta a profundizar los servicios financieros en mercados no tradicionales.

Sin mencionar las ventajas de permitir un desarrollo financiero local y más cercano a las regiones rurales del país, pero también cercano a las personas, con un menor riesgo de evasión de impuesto y de fuga de capitales. El auspicio también de iniciativas financieras comunitarias a menor escala como las cajas de ahorro, monedas sociales o comunidades de intercambio pueden favorecer la estabilidad social del país y no solo la de la moneda fiat, además permite disputar el monopolio bancario a las entidades financieras concentradas, lo que puede ser 
Figura 4. Bancos privados: destino del crédito 2002-2018

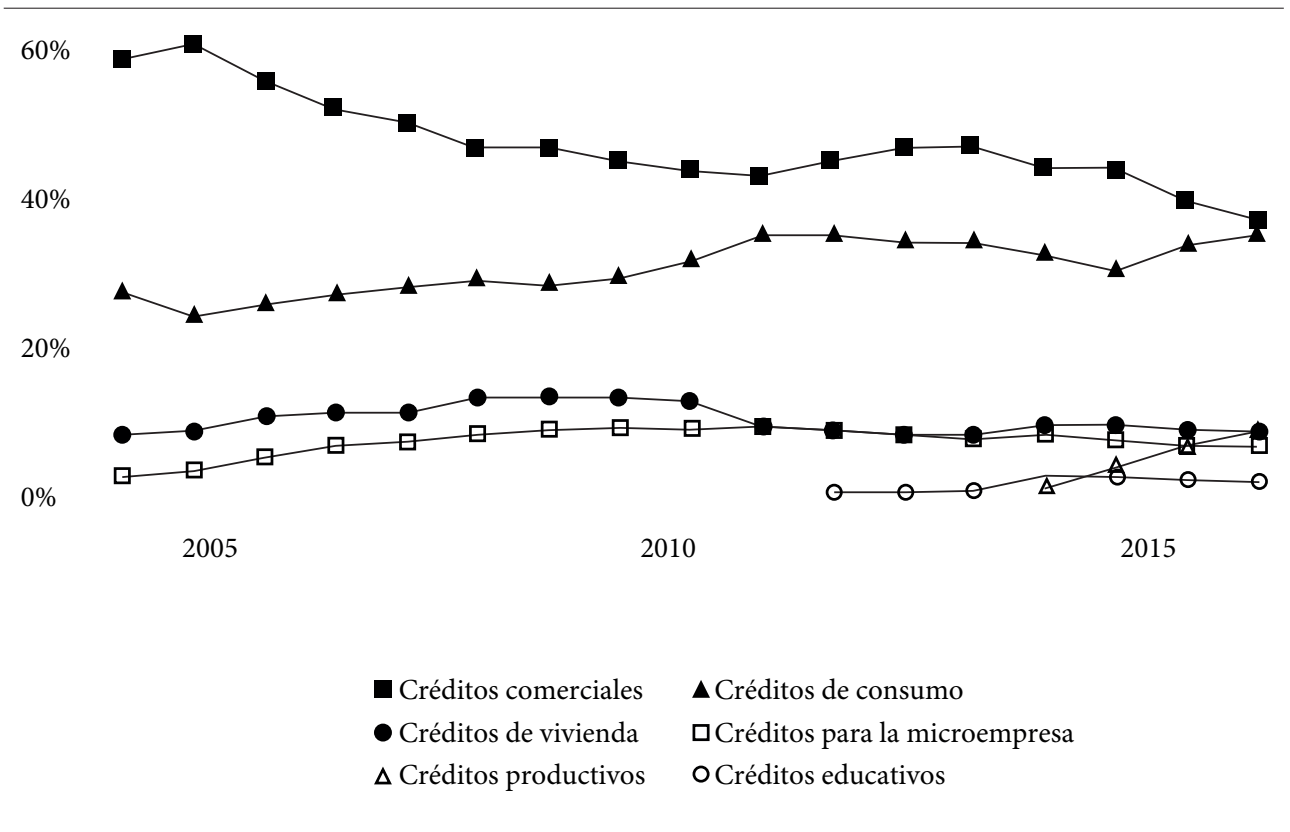

Fuente: Superintendencia de Bancos del Ecuador.

aún más eficiente, sin tener que sacrificar las leyes en los altares de la competencia neoclásica. Los créditos de consumo deberían ser por definición más riesgosos, por lo que se puede decir que los bancos privados prefieren otorgar créditos de consumo en las provincias de Pichincha, Guayas y Azuay, créditos cuyo acceso además está reservado a estratos altos de ingreso, nos lleva a plantearnos la pregunta ¿hasta qué punto los bancos privados auspician la inversión productiva en el país?

\section{NOTAS}

1 El trabajo de Minsky en cuanto al sector financiero se centra en la idea de que el sector financiero es inherentemente susceptible a escenarios de especulación que pueden llevar a crisis, lo que quiere decir que la crisis sería inherente al sistema financiero.

2 Estudio realizado midiendo el efecto de las desregulaciones financieras en la Unión Europea.

3 En este estudio, el autor utilizó el porcentaje de la participación de las tres mayores empresas del mercado, el mismo índice para las cinco mayores y el índice Herfindahl-Hirschman

4 Para el estudio, también se utilizó el índice de Gini para explorar el uso de este indicador en el estudio de la concentración, los resultados con el índice de Gini fueron diferentes debido a la naturaleza matemática del indicador, pero en ningún caso resultaron ser contradictorios, se decidió no incluir estos resultados en el documento para no redundar más en los resultados.

\section{REFERENCIAS}

Asobanca. (2019). Informe Técnico: Tasas de interés. Publicaciones Asociación de Bancos del Ecuador. 
Beck, T. (2008). Bank competition and Financial stability: Friends or Foes? Policy Research working paper. Camino, S., Uzcátegui, C. y Moran, J. (2017). Estructura de mercado del sistema bancario ecuatoriano: concentración y poder de mercado. Cumbres, o9-16.

Casu, B. and Girardone, C. (2006). Bank Competition, Concentration and Efficiency in the Single European Market. Manchester School, 441-468.

Cetorelli, N. (2006). Real effects of Bank competition. The Ohio State University Press, 543-558.

DeYoung, R. and Rice, T. (2004). Noninterest income and financial performance at US commercial banks. Financial Review, 101-127.

Durand, F. (2020). El debate sobre la captura del Estado en América Latina y la vuelta del instrumentalismo, con referencia especial a Perú. En Concentración económica y poder político en América Latina (pp. 44-92). Buenos Aires.

Hernández, I. (2010). Tributación y desarrollo en perspectiva. Revista de economía institucional, 13-24. Jaramillo, O. y Ramírez, R. (2020). Concentración y dinámica empresarial: Estudio estructural de la economía ecuatoriana. Repositorio Universidad Central del Ecuador, Facultad de Ciencias Económicas.

King, R. and Levine, R. (1993). Finance, entrepreneurship and growth. Theory and evidence. Journal of Monetary Economics, 513-542.

Levine, R. (1997). Financial development and economic growth: views and agenda. Journal of money, credit and banking, 596-613.

Marichal, C. y Gambi, T. (2017). Historia bancaria y monetaria de América Latina (siglos xIx y xx). Editorial de la Universidad de Cantabria.

Marx, K. (1978). Capital. Crítica de la Economía Política.

Minsky, H. (2008). Stabilizing an unstable economy. McGraw Hill.

Miño Grijalva, W. (2008). Breve Historia Bancaria del Ecuador. Corporación Editora Nacional.

Morandé, F. (2016). A casi tres décadas del Consenso de Washington ¿Cuál es su legado en América Latina? (s. d.).

Pepall, L., Richards, D. and Norman, G. (2014). Industrial organization contemporary theory and empirical applications $\left(5^{\text {th }}\right.$ ed.). Wiley.

Pozo, S. (2018). Competencia y estabilidad en el sistema bancario: Evidencia empírica para el Ecuador durante el periodo 2001-2016. Universidad de Cuenca .

Regalado, P. y Espinoza, V. (2018). Desarrollo financiero en Ecuador: Análisis de la concentración del sector cooperativo de ahorro y crédito del Segmento 1. X-Pedientes económicos, 20-36.

Rochet, J.-C. and Freixas, X. (1999). Microeconomics of Banking. MIT Press.

Schumpeter, J. (1997). Teoría del desenvolmiento económico. Fondo de Cultura Económica.

Vera, C. y Titelman, D. (2013). El sistema financiero en América Latina y el Caribe. Naciones Unidas.

Vera-Gilces, P., Camino-Mogro, S., Ordeñana-Rodríguez, X. and Cornejo-Marcos, G. (2019). A look inside banking profitability: Evidence from a dollarized emerging country. The Quarterly Review of Economics and Finance. (s. d.).

Weeks, J. (2009). Teoría de la competencia en los neoclásico y en Marx. MAIA Ediciones. 